\title{
Computer-aided analysis of in-flight physiological measurement
}

\author{
GERALD C. ARMSTRONG \\ Computer Science Corporation, U.S. Air Force Flight Test Center, Edwards Air Force Base, California
}

Heart waveforms were collected from pilots under laboratory baseline conditions and in flight. Described is the computer-aided method for smoothing the heart waveforms recorded in flight.

For many years, psychologists and physiologists have been developing methods to assess mental and physical workload. Reid, Shingledecker, and Eggemeier (1981) introduced the Subjective Workload Assessment Technique (SWAT), the application of conjoint measurement to workload scale development. SWAT gives the analyst a tool to assess task-load time pressure, mental effort, and psychological stress. SWAT has been used in several Air Force simulator studies and flight tests.

Psychologists at the Air Force Flight Test Center (AFFTC) have been working with physiologists at the Air Force School of Aerospace Medicine to develop methods to obtain objective measures of pilot workload. A computer-aided analysis (PAWAN) has been developed to assess pilot control activity, control error rate, performance level, and heart rate variation.

SWAT and PAWAN were used together for the first time at AFFTC, during the A-10 Single Seat Night Attack flight test program to assess the merits of each technique. The results were reported by Mead, Hudson, and Armstrong (1984) in an Air Force technical report. It was found that more development was required in both SWAT and PAWAN to eliminate problems experienced in data collection in an operational flight test environment. The purpose of this paper is to present the difficulties encountered in obtaining in-flight heart rate data and describe the computer-aided techniques used to overcome the problems.

\section{BACKGROUND}

A primary human factor objective of the A-10 singleseat night-attack flight test program was to determine whether a correlation existed between a pilot's subjective assessment of workload using Subjective Workload Assessment Technique (SWAT) and objective measures of workload using PAWAN. The low-level night-attack profiles flown over level, rolling, and mountainous terrain in clear air and high-humidity conditions produced a wide range of pilot workload. However, problems were en-

The author's mailing address is: Computer Science Corporation, U.S. Air Force Flight Test Center, Edwards Air Force Base, CA 93523. countered in obtaining the in-flight subjective assessments (SWAT scores) and in analyzing the pilot's heart rate. As the flight conditions went from benign to hostile, the pilot's stress and workload increased to the point that he could not give sufficient attention to the SWAT scores. His attention was concentrated on keeping the aircraft at a safe but close ground-clearance altitude and on searching the heads-up display infrared video to clear flight obstructions and find targets. During times of lower workload, there were numerous radio transmission interruptions from the forward air controller's briefing on the next target. The pilot was not able to return to reporting SWAT scores due to the interfering operational duties of setting up for the next attack.

The difficulties in analyzing the heart rate data were due to electronic interference noise superimposed on the sensitive waveform signal. Other PAWAN parameters (longitudinal stick control and radar altitude performance) were clear of noise. Several filtering methods were tested to remove the interference noise prior to recording the heart waveform, but none were effective, since the electrocardiogram (ECG) signal required amplification prior to recording. The option was taken to smooth the recorded heart waveform during analysis. This was accomplished by computer-aided methods and techniques.

\section{PROCEDURE AND EVALUATION}

Prior to flight testing, baseline heart waveforms were obtained on the pilots using the same signal-conditioning unit used during the flights. For the tests, the pilots wore three chest-mounted ECG sensors during baseline recording.

The heart waveform analog signal was fed from the conditioning unit into a programmable digital oscilloscope. A mainframe oscilloscope (Tektronix 7854) with waveform calculator was used to combine the features of a highperformance oscilloscope with a waveform-oriented, dedicated-function digital processor. Additional plug-in units provided time base with pretrigger acquire clock (Tektronix 7B87), delaying time base (Tektronix 7B25), and dual trace amplifier (Tektronix 7A26). These features provided all standard waveform analysis capabilities, including smoothing functions and provisions for waveform 
digitizing at repeatable $\mathrm{R}$-wave triggering magnitudes.

As many as 10 heart waveforms could be stored in the programmable oscilloscope memory, and provisions were available to display both real-time waveforms and stored waveforms for direct comparison and measurement. The oscilloscope program provided automatic computercontrolled digitizing of randomly selected waveforms. The oscilloscope was controlled remotely over a generalpurpose interface bus connected to a graphic computer (Tektronix 4054).

During baseline recording, the pilot's heart waveforms were recorded randomly under the following conditions: (1) seated, at rest; (2) standing exercise, running in place; and (3) seated activity, eye/hand coordination, and mental problem solving. These baseline recordings provided a full range of waveforms from low beats per minute (BPM) to high BPM.

Figure 1 shows a typical baseline waveform after oscilloscope smoothing and computer processing. The occurrences of $\mathrm{P}$-wave, $\mathrm{R}$-wave, and T-wave are indicated on the baseline waveform. Essentially, the smoothing process replaced each point of the stored waveform with

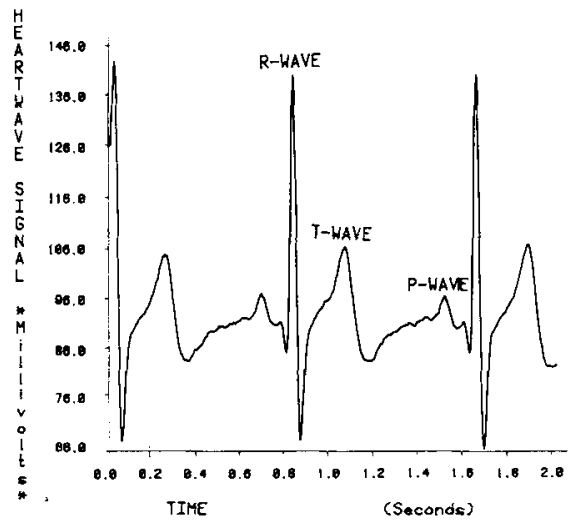

Figure 1. Baseline heart waveform.

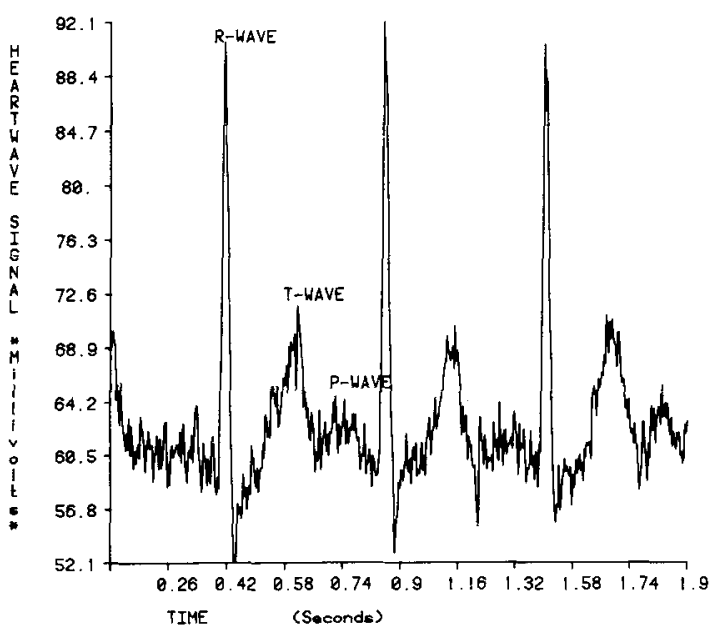

Figure 2. Unsmoothed in-flight waveform.

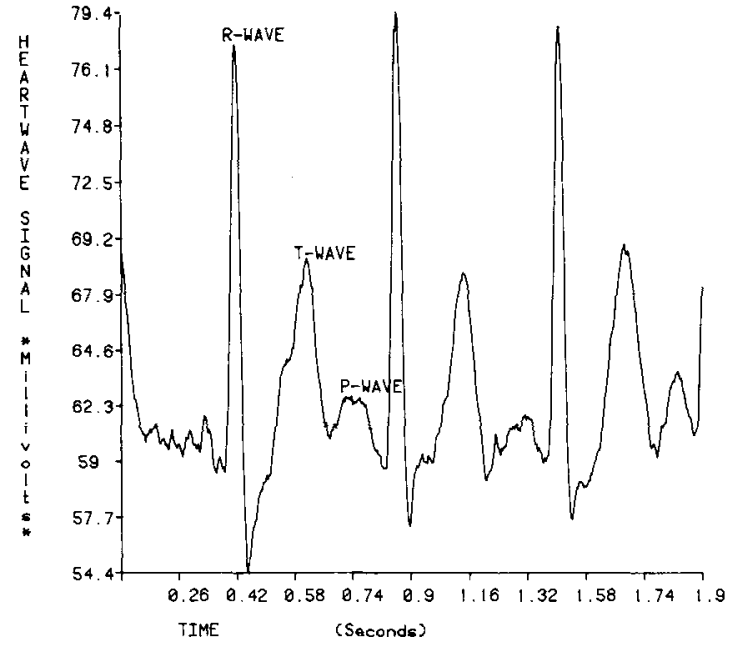

Figure 3. Smoothed in-flight waveform.

the average value of the waveform within the specified distance about the point. The computer-controlled, programmable oscilloscope assured that each baseline and in-flight heart waveform received the same magnitude of smoothing. In every case, the point-to-point average was used to smooth and replace each point in the waveform.

Figure 2 shows a typical heart waveform that was recorded in flight. The ECG analog signal was sampled at 250 times per second and recorded in analog form on the flight test tape system. The tape-stored waveform was then digitized at 250 times per second and stored in a CYBER-74 computer. The digitized values were transferred by interface modem to the graphic computer. Next, the analog waveform was fed into the digital oscilloscope for computer-controlled smoothing. The waveform shown in Figure 2 shows the interference noise superimposed on the waveform prior to smoothing.

Figure 3 shows the waveform after smoothing and after being fed back to the graphic computer for plotting. Figure 3 shows the waveform immediately after smoothing to indicate the effectiveness of the smoothing process.

The waveform shown in Figure 1 was recorded in a laboratory-like environment after being directly digitized from the signal-conditioning unit into the oscilloscope. However, these digitized values were stored in the CYBER-74, having been passed by telephone modem from the graphic computer. The waveform values were passed back again from the CYBER-74 to the graphic computer prior to the plotting shown in Figure 1. The Figure 1 waveform did not undergo the analog tape recording as did the waveforms in Figure 2 and Figure 3. Figure 1 waveform also did not undergo the same digitizing process as the waveforms in Figure 2 and Figure 3.

\section{CONCLUSIONS}

It is expected that, in the future, baseline recordings will be recorded in the aircraft with all electrical systems functioning to assure that all waveforms receive the same 
treatment on the way to final analysis. Nonetheless, the value of computer-aided analysis on in-flight physiological measurements was shown by the results of this flight test effort. The basic fact that the occurrences of P-wave, $R$-wave, and T-wave were detected on the in-flight waveform was felt to be a step in the right direction toward obtaining objective measures of workload.

\section{REFERENCES}

Mead, T. U., Hudson, J. C., \& Armstrong, G. C. (1984). Single seat night attack A-10 follow-on (Phase $I I$ ) (AFFTC-TR-84-5). Edwards Air Force Base, CA: Air Force Flight Test Center. (Secret) Reid, G. B., Shingledecker, C. A., \& EGgemeier, F. T. (1981). Application of conjoint measurement to workload scale development. Paper presented at the 25th annual meeting of the Human Factors Society, Rochester, NY. 